\title{
A Survey of Fuzzy Convex Programming Models
}

\author{
Ricardo C. Silva, Carlos Cruz, José L. Verdegay, and Akebo Yamakami
}

\begin{abstract}
Optimization is a procedure of finding and comparing feasible solutions until no better solution can be found. It can be divided into several fields, one of which is the Convex Optimization. It is characterized by a convex objective function and convex constraint functions over a convex set which is the set of the decision variables. This can be viewed, on the one hand, as a particular case of nonlinear programming and, on the other hand, as a general case of linear programming. Convex optimization has applications in a wide range of real-world applications, whose data often cannot be formulate precisely. Hence it makes perfect sense to apply fuzzy set theory as a way to mathematically describe this vagueness. In this paper we review the theory about this topic and describe some flexible and possibilistic programming models to solve fuzzy convex programming problems. Flexible programming uses fuzzy sets to represent the vagueness of the decision maker's aspirations and constraints, while possibilistic programming models imprecise or ambiguous data by possibility distributions.
\end{abstract}

\section{Introduction}

Mathematical programming is used to solve problems, achieving the best outcome of the objective function in a function domain that can be constrained or not. This kind of problem is called an optimization problem or

Carlos Cruz · José L. Verdegay

Department of Computer Science and Artificial Intelligence, University of Granada, E-18071, Granada, Spain

e-mail: \{carloscruz, verdegay\}@decsai.ugr.es

Ricardo C. Silva · Akebo Yamakami

Department of Telematics, School of Electrical and Computer Engineering, P.O.

Box 6101, University of Campinas, 13083-970, Campinas, SP, Brazil

e-mail: \{rcoelhos, akebo\}@dt.fee.unicamp.br 
a mathematical programming problem, in which the aim is to find the best of all possible solutions. More formally, find a solution in the feasible region which has the minimum (or maximum) value of the objective function. If all the functions are linear, we obviously have a linear program. Otherwise, the problem is called a nonlinear program. However, many realistic problems cannot be adequately represented or approximated as a linear program owing to the nature of the non-linearity of the objective function and/or the non-linearity of any of the constraints. As it is well known Convex Programming represents a special class of mathematical programming in which the objective function is convex and the set of constraints are formed by convex functions over a convex decision space.

Thus, on the one hand, it is clear that convex programming encompasses all linear programming problems, including applications in scheduling, planning and flow computations, and they may be used to solve some interesting combinatorial optimization problems. On the other hand, it can be viewed as a particular case of nonlinear programming and it is more general than quadratic programming. Nowadays we can use highly efficient and robust algorithms and software for convex programming which are important tools for solving problems in diverse fields. However in many real practical applications one lacks of exact knowledge [14, and only approximate, vague and imprecise values are known. Experience shows that the best way of modeling these kinds of problems is using Soft Computing methodologies [33.

In recent years, Soft Computing, and Fuzzy Logic in particular, has shown great potential for modeling systems which are non-linear, complex, illdefined and not well understood. Fuzzy Logic has found numerous and different applications due to its easy implementation, flexibility, tolerant nature to imprecise data, low cost implementations and ability to model non-linear behavior of arbitrary complexity because of its basis in terms of natural language.

In the fuzzy environment, as it happens in the case of linear programming problems, a variety of fuzzy convex programming problems can be defined: Convex programming problems with a fuzzy objective, i.e., with fuzzy numbers defining the costs of the objective function, convex programming problems with a fuzzy goal, i.e., with some fuzzy value to be attained in the objective, convex programming problems with fuzzy numbers defining the coefficients of the technological matrix and, finally, with a fuzzy constraint set, i.e., with a feasible set defined by fuzzy constraints.

Thus, fuzzy convex programming is applied in a wide range of disciplines, such as: control systems problems [13, 21, 29], production planning and scheduling problems in the complex industrial systems [26, 27], modeling multi-product aggregate production planning (APP) problems with fuzzy demands and fuzzy capacities [28, regression models [8, 25, 7], portfolio selection problem [1, 12, 16, 24, 31, 35, 34. Some others interesting papers where various authors apply soft computing methodologies to convex programming are [2, 15, 17, 23, 26, 36. 
With this in mind, the objective of this paper is to review the theory about this topic and to describe some flexible and possibilistic approaches to solve fuzzy convex programming problems remarking some limitations in their formulations.

The paper is organized as follows: Section 2 shows how is formulated a convex programming problem and what problems belong to it; Section 3 presents the formulation of a convex programming problem under fuzzy environment and describes some known flexible and possibilistic methods that were developed to solve fuzzy convex programming problems with uncertainties in the relationships or coefficients. In Section 4 is described a approach that solves convex programming problems with uncertainties in the relationships and an numerical example is solved by using this appraoch. Finally, conclusions are presented in Section 5 .

\section{Convex Programming}

Some real-world problems can be formulated as mathematical programming problems that find to obtain the best solution according to the situation to be solved. This problems can have one or several objectives over a set of constraints or not, but only the problems with one objective will be described in this work. Then, these mathematical programming problems can be written as

$$
\begin{array}{ll}
\min & f_{0}(\mathbf{x}) \\
\text { s.t. } & f_{i}(\mathbf{x}) \leq \mathbf{0} i=1, \ldots, m \\
& \mathbf{x} \in \Omega .
\end{array}
$$

where $\mathbf{x}$ is a decision variables vector, $\Omega$ is the feasible solutions convex set in $\mathbb{R}^{n}, f_{0}: \mathbb{R}^{n} \rightarrow \mathbb{R}$ is the objective or cost function, and $f_{i}: \mathbb{R}^{n} \rightarrow \mathbb{R}$, for each $i=1, \ldots, m$, are constraint functions.

Nevertheless, a convex optimization problem is one in which the objective function, $f_{0}$, is convex, and the feasible solution set formed by the constraints, $f_{i}(i=1, \ldots, m)$, if any, form a convex set [5, 4]; i.e. the function satisfies the following equation:

$$
f(\alpha \mathbf{x}+(1-\alpha) \mathbf{y}) \leq \alpha f(\mathbf{x})+(1-\alpha) f(\mathbf{y})
$$

for all $\mathbf{x}, \mathbf{y} \in \mathbb{R}^{n}$ and all $\alpha \in[0,1]$.

According to the definition of convex optimization, it is easy to see that the linear programming problems belong to this kind of problems. There are many other problems that belong to the set of convex programming problems but we can highlight the quadratic programming problems that are most important in a great field of real-world problems and defined how a quadratic problem 
if the objective function is quadratic and constraint functions are linear. Thus, Problem (1) can be rewritten as a quadratic programming problem in the following way:

$$
\begin{aligned}
& \min z=f_{0}(\mathbf{c}, \mathbf{x})=\mathbf{c}^{\mathbf{t}} \mathbf{x}+\frac{1}{2} \mathbf{x}^{\mathbf{t}} \mathbf{Q} \mathbf{x} \\
& \text { s.t. } \mathbf{f}(\mathbf{A}, \mathbf{x})=\mathbf{A x} \leq \mathbf{b} \\
& \quad \mathbf{x} \leq 0
\end{aligned}
$$

where $\mathbf{c}$ is the $n$-dimensional vector and $\mathbf{Q}$ is the $n \times n$-dimensional matrix of profit coefficients of the objective function, $\mathbf{b}$ is the $m$-dimensional vector of total resources available, $\mathbf{A}$ is the matrix of technical coefficients, and $\mathbf{x}$ is the $n$-dimensional vector of decision variables (or alternatives).

If an optimization problem can be formulated as a convex optimization problem, then it is solved efficiently.

\section{Fuzzy Convex Programming}

Similar as the case of fuzzy linear programming problems 15, a large number of fuzzy convex programming problems can be defined. The uncertainties can be found in the relationships, constants, decision variables or in all parameters of the problem. In contrast to fuzzy linear programming problems where much research has been conducted, unfortunately, little has been done with this important class of problems.

A conventional programming problem (1) can be transformed into a fuzzy convex formulation with vagueness in all their parameters as:

$$
\begin{array}{ll}
\widetilde{\min } & f_{0}(\tilde{\mathbf{c}} ; \tilde{\mathbf{x}}) \\
\text { s.t. } & f_{i}(\tilde{\mathbf{a}} ; \tilde{\mathbf{x}}) \lesssim \tilde{\mathbf{0}} i=1, \ldots, m \\
& \tilde{\mathbf{x}} \in \tilde{\Omega} .
\end{array}
$$

where $\tilde{\mathbf{x}}$ is the fuzzy variables decision vector, $\tilde{\Omega}$ is the fuzzy feasible solutions set, $\tilde{\mathbf{c}}$ is the fuzzy cost vector, $\tilde{\mathbf{a}}$ is the fuzzy coefficients vector of the constraint functions, and $\lesssim$ is the fuzzy relation.

Each fuzzy parameter in Problem (3) can be defined by membership functions such as: $\mu_{i}: \mathbb{R} \rightarrow[0,1], i=1, \ldots, m$.

According to a general classification of fuzzy mathematical programming into flexible programming [12, 37] and possibilistic programming [10, 11, 22] several methods to solve convex programming will be presented in next subsections. The distinction between flexible (fuzzy) programming and possibilistic programming is developed in . Flexible programming uses fuzzy sets to represent the vagueness of the decision maker's aspirations and constraints and possibilistic programming models imprecise or ambiguous data by possibility distributions. 


\subsection{Flexible Convex Programming}

In this subsection we will describe some approaches that use flexible optimization to solve fuzzy convex programming problems. The uncertainties can be found in the costs of the objective function and/or constants of the constraint functions of the problem.

\subsubsection{Tang and Wang Approach}

Tang and Wang [26, 27] proposed two methods to solve the quadratic programming problems. They study quadratic programming problems with a type of fuzzy objective and resource constraints and its solution method: an interactive approach. Also, they focus on a non-symmetric model for fuzzy nonlinear programming problems with penalty coefficients and attempts to develop a systematic approach to solve them. It uses a kind of nonlinear membership function to describe the fuzzy available resources and fuzzy constraints.

Hence, quadratic programming problems with uncertainty in the vector of the independent coefficients $\tilde{\mathbf{b}}$ is defined by Tang and Wang in the following way:

$$
\begin{gathered}
\widetilde{\min } \mathbf{c}^{\mathbf{t}} \mathbf{x}+\frac{1}{2} \mathbf{x}^{\mathbf{t}} \mathbf{Q x} \\
\text { s.t. } \mathbf{A x} \leq \tilde{\mathbf{b}} \\
\mathbf{x} \in \Omega .
\end{gathered}
$$

A membership function for each fuzzy component of vector $\tilde{\mathbf{b}}$ is needed to solve this problem:

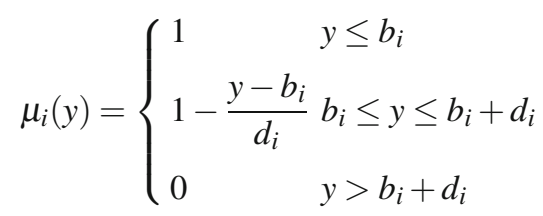

where $d_{i},(i=1, \ldots, m)$ is the allowed maximum tolerance in each restriction. Similar as extended Zimmermann's approach [2] the membership function can be transformed as:

$\max \alpha$

$$
\begin{array}{ll}
\text { s.t. } & \mu_{0}(\mathbf{x}) \leq \alpha \\
& \mu_{i}(\mathbf{x}) \leq \alpha, i=1, \ldots, m \\
& \mathbf{x} \in \Omega, \alpha \in[0,1] .
\end{array}
$$


or

max Target

$$
\begin{array}{ll}
\text { s.t. } & \mu_{0}(\mathbf{x}) \leq \alpha_{0} \\
& \mu_{i}(\mathbf{x}) \leq \alpha_{0}, i=1, \ldots, m \\
& \mathbf{x} \in \Omega, \alpha_{0} \in[0,1] .
\end{array}
$$

where $\alpha_{0}$ is a satisfaction degree that is in the interval $[0,1]$. This degree is an acceptable value chosen by the decision maker. Target can be an objective function, restrictions or another goal given by the decision maker.

According to described idea in [26, 27] an optimal solution can be defined as:

Definition 1. A fuzzy optimal solution of Problem (4) is a fuzzy set $\tilde{S}$ defined by

$$
\tilde{S}=\left\{\left(\mathbf{x}, \mu_{\tilde{S}}(\mathbf{x})\right) \mid \mathbf{x} \in \Omega\right\}
$$

with

$$
\mu_{\tilde{S}}(\mathbf{x})=\min \left\{\mu_{0}(\mathbf{x}), \mu_{1}(\mathbf{x}), \ldots, \mu_{m}(\mathbf{x})\right\}
$$

If

$$
S_{\alpha}=\left\{\mathbf{x} \in \Omega \mid \mu_{\tilde{S}}(\mathbf{x}) \geq \alpha\right\},
$$

where $\alpha \in[0,1]$. Then $S_{\alpha}$ is a set with cutting level $\geq \alpha$ of $\tilde{S}$.

Definition 2. $\alpha^{*}$ is the best satisfaction level if there exists an $\alpha \in[0,1]$, such that $\forall 0 \leq \alpha<\alpha^{*}, S_{\alpha}$ is non-empty and, $\forall \alpha \geq \alpha^{*}, S_{\alpha}$ is empty.

This approach uses a classic inequality concept to compare kind of different numbers. Thus, it is limited because this approach formulates a comparison of fuzzy numbers, vector of right-hand sides, with a crisp matrix of constraint coefficients.

\subsubsection{Liu Approach}

An approach to solve quadratic programming problems with fuzzy costs and fuzzy coefficients in the restrictions set was proposed by Liu [17, 18]. Vector constants $\mathbf{c}$ and $\mathbf{b}$, and the matrix $\mathbf{A}$ are uncertainties. Thus, Problem (2) can be transformed into fuzzy programming problem in the following form:

$$
\begin{gathered}
\min \tilde{\mathbf{c}} \mathbf{x}+\frac{\mathbf{1}}{\mathbf{2}} \mathbf{x}^{\mathbf{t}} \mathbf{Q} \mathbf{x} \\
\text { s.t. } \tilde{\mathbf{A}} \mathbf{x} \leq \tilde{\mathbf{b}} \\
\mathbf{x} \in \Omega .
\end{gathered}
$$


where $\tilde{\mathbf{c}}=\left\{\left(c_{j}, \mu_{\tilde{c}_{j}}\left(c_{j}\right)\right), \quad j=1, \ldots, n \mid c_{j} \in \operatorname{supp}\left(\tilde{c}_{j}\right)\right\}, \tilde{\mathbf{A}}=\left\{\left(a_{i j}, \mu_{\tilde{a}_{i j}}\left(a_{i j}\right)\right), \quad i=\right.$ $1, \ldots, m$ y $\left.j=1, \ldots, n \mid a_{i j} \in \operatorname{supp}\left(\tilde{a}_{i j}\right)\right\}$ and $\tilde{\mathbf{b}}=\left\{\left(b_{i}, \mu_{\tilde{b}_{i}}\left(b_{i}\right)\right), i=1, \ldots, m \mid b_{i} \in\right.$ $\left.\operatorname{supp}\left(\tilde{b}_{i}\right)\right\}$.

The authors derive the membership function of the fuzzy goal, and then they apply Zadeh's extension principle to transform the fuzzy quadratic problem into a pair of two-level mathematical programs to calculate the upper and lower bounds of the objective value at possibility level. These programs can be solved by conventional optimization techniques. Thus, the membership function of the objective function can be defined as

$$
\mu_{\tilde{Z}}(z)=\sup _{\mathbf{c}, \mathbf{A}, \mathbf{b}} \min \left\{\mu_{\tilde{c}_{j}}\left(c_{j}\right), \mu_{\tilde{a}_{i j}}\left(a_{i j}\right), \mu_{\tilde{b}_{i}}\left(b_{i}\right), \forall i, j \mid z=Z(\mathbf{c}, \mathbf{A}, \mathbf{b})\right\}
$$

where $Z(\mathbf{c}, \mathbf{A}, \mathbf{b})$ is the function of the conventional quadratic problem (3). Membership function $\mu_{\tilde{Z}}$ can be computed by finding the functions that describe the shape of the left and right sides of the fuzzy numbers. Then, it is possible to obtain the upper bound of the objective value $Z_{\alpha}^{U}$ and the lower bound $Z_{\alpha}^{L}$ to each value $\alpha$. Thus, $Z_{\alpha}^{U}$ is the maximum and $Z_{\alpha}^{L}$ is the minimum of $Z(\mathbf{c}, \mathbf{A}, \mathbf{b})$, respectively, that can be described as:

$$
\begin{aligned}
Z_{\alpha}^{U} & =\max \{Z(\mathbf{c}, \mathbf{A}, \mathbf{b})\} \\
Z_{\alpha}^{L} & =\min \{Z(\mathbf{c}, \mathbf{A}, \mathbf{b})\}
\end{aligned}
$$

where each component $j$ of the vector $\mathbf{c}$ belong to the interval $\left[\left(c_{j}\right)_{\alpha}^{L},\left(c_{j}\right)_{\alpha}^{U}\right]$, each components $i j$ of the matrix $\mathbf{A}$ belong to the interval $\left[\left(a_{i j}\right)_{\alpha}^{L},\left(a_{i j}\right)_{\alpha}^{U}\right]$, and each component $i$ of the vector $\mathbf{b}$ belong to the interval $\left[\left(b_{i}\right)_{\alpha}^{L},\left(b_{i}\right)_{\alpha}^{U}\right]$, for all $i=1, \ldots, m$ and $j=1, \ldots, n$.

Different values of fuzzy parameters produce different objective values, then equations (9) and (10) can transform the fuzzy quadratic problem into two levels.

Using equation (9) the fuzzy problem can be transformed as:

$$
\begin{gathered}
\max \\
Z_{\alpha}^{U}=\begin{array}{c}
\left(c_{j}\right)_{\alpha}^{L} \leq c_{j} \leq\left(c_{j}\right)_{\alpha}^{U} \\
\left(a_{i j}\right)_{\alpha}^{L} \leq a_{i j} \leq\left(a_{i j}\right)_{\alpha}^{U} \\
\left(b_{i}\right)_{\alpha}^{L} \leq b_{i} \leq\left(b_{i}\right)_{\alpha}
\end{array}\left\{\begin{aligned}
& \min _{\mathbf{x}} \sum_{j=1}^{n} c_{j} x_{j}+\frac{1}{2} \sum_{j=1}^{n} \sum_{l=1}^{n} q_{j l} x_{j} x_{l} \\
& \mathrm{s.t.} \sum_{j=1}^{n} a_{i j} x_{j} \leq b_{i}, \quad i=1, \ldots, m \\
& \mathbf{x} \in \Omega
\end{aligned}\right. \\
\end{gathered}
$$

where goal value $Z_{\alpha}^{U}$ is the upper bound of the classical quadratic programming problem.

Using equation (10) the fuzzy problem can be transformed as:

$$
\begin{gathered}
\min \\
Z_{\alpha}^{L}=\begin{array}{c}
\left(c_{j}\right)_{\alpha}^{L} \leq c_{j} \leq\left(c_{j}\right)_{\alpha}^{U} \\
\left(a_{i j}\right)_{\alpha}^{L} \leq a_{i j} \leq\left(a_{i j}\right)_{\alpha}^{U} \\
\left(b_{i}\right)_{\alpha}^{L} \leq b_{i} \leq\left(b_{i}\right)_{\alpha}
\end{array}
\end{gathered}\left\{\begin{aligned}
& \min _{\mathbf{x}} \sum_{j=1}^{n} c_{j} x_{j}+\frac{1}{2} \sum_{j=1}^{n} \sum_{k=1}^{n} q_{j k} x_{j} x_{k} \\
& \text { s.t. } \sum_{j=1}^{n} a_{i j} x_{j} \leq b_{i}, \quad i=1, \ldots, m \\
& \mathbf{x} \in \Omega
\end{aligned}\right.
$$


where goal value $Z_{\alpha}^{L}$ is the lower bound of the classical quadratic programming problem.

These two formulations above need two programs to solve them called outer-level and inner-level programs. Outer-level program obtains the values $c_{j}, a_{i j}$ and $b_{i}$ that are used with parameters by inner-level program. Innerlevel program solves a classical quadratic programming problem with the data obtained by outer-level program. The authors state that the formulation of two-level quadratic problems is a generalization of the conventional parametric quadratic programming problem.

Thus, firstly, the two-level mathematical program is transformed into the following quadratic problem by dual formulation:

$$
\begin{aligned}
\max & -\frac{1}{2} \sum_{i=1}^{n} \sum_{j=1}^{n} h_{i j} x_{i} x_{j}-\sum_{i=1}^{m}\left(b_{i}\right)_{\alpha}^{U} \lambda_{i} \\
\text { s.t. } & \sum_{i=1}^{n} h_{i j} x_{i}+\sum_{i=1}^{m} a_{i j} \lambda_{i}-\delta_{j}=-c_{j}, \quad j=1,2, \ldots, n \\
& \left(c_{j}\right)_{\alpha}^{L} \leq c_{j} \leq\left(c_{j}\right)_{\alpha}^{U}, \quad j=1,2, \ldots, n \\
& \left(a_{i j}\right)_{\alpha}^{L} \leq a_{i j} \leq\left(a_{i j}\right)_{\alpha}^{U}, \quad i=1,2, \ldots, m, \quad j=1,2, \ldots, n \\
& \lambda_{i}, \delta_{j} \geq 0, \quad i=1,2, \ldots, m \text { and } j=1,2, \ldots, n .
\end{aligned}
$$

Since both the inner-level program and outer-level of the second program have the same minimization operation, they can be combined into a conventional one-level program with the constraints of the two programs considered simultaneously. Consequently, some points must be analyzed which are shown in [17, 18. The second program can be described as:

$$
\begin{aligned}
\max & \sum_{j=1}^{n}\left(c_{j}\right)_{\alpha}^{L}+\frac{1}{2} \sum_{i=1}^{n} \sum_{j=1}^{n} h_{i j} x_{i} x_{j} \\
\text { s.t. } & \sum_{i=1}^{n}\left(a_{i j}\right)_{\alpha}^{L} x_{i} \leq\left(b_{i}\right)_{\alpha}^{U}, \quad i=1,2, \ldots, m \\
& x_{j} \geq 0, \quad j=1,2, \ldots, n
\end{aligned}
$$

This approach is limited because it uses in their formulation a crisp inequality to compare fuzzy numbers, vector of right-hand sides, with a fuzzy matrix of constraint coefficients.

\subsubsection{Ammar and Khalifa Approach}

An approach to solve quadratic programming problem with fuzzy costs $\tilde{Q}$, fuzzy matrix coefficients $\tilde{A}$ and the restrictions set vector $\tilde{b}$ was proposed in [1. Thus, Problem (2) can be defined in the following form:

$$
\begin{gathered}
\min \mathbf{x}^{\mathbf{t}} \tilde{\mathbf{Q}} \mathbf{x} \\
\text { s.t. } \tilde{\mathbf{A}} \mathbf{x} \leq \tilde{\mathbf{b}} \\
\mathbf{x} \in \Omega .
\end{gathered}
$$

where all decision variables are non-negative 
Problem (15), can be defined in $\alpha$-cut intervals as:

$$
\begin{gathered}
\left(P_{\alpha}\right): \min \mathbf{x}^{\mathbf{t}}\left[\mathbf{Q}_{\alpha}^{\mathbf{L}}, \mathbf{Q}_{\alpha}^{\mathbf{U}}\right] \mathbf{x} \\
\text { s.t. }\left[\mathbf{A}_{\alpha}^{\mathbf{L}}, \mathbf{A}_{\alpha}^{\mathbf{U}}\right] \mathbf{x} \leq\left[\mathbf{b}_{\alpha}^{\mathbf{L}}, \mathbf{b}_{\alpha}^{\mathbf{U}}\right] \\
\mathbf{x} \in \Omega .
\end{gathered}
$$

where all decision variables are non-negative and $\alpha \in(0,1]$.

Problem (16) can be divided into two classic problems. Using the lower bound of the interval of $\alpha$-cuts for the first problem and using the upper bound for the second we can transform this problem as:

$$
\begin{gathered}
\left(P_{\alpha}^{L}\right): \min \mathbf{x}^{\mathbf{t}} \mathbf{Q}_{\alpha}^{\mathbf{L}} \mathbf{x} \\
\text { s.t. } \mathbf{A}_{\alpha}^{\mathbf{L}} \mathbf{x} \leq \mathbf{b}_{\alpha}^{\mathbf{L}} \\
\mathbf{x} \in \Omega . \\
\left(P_{\alpha}^{U}\right): \min \mathbf{x}^{\mathbf{t}} \mathbf{Q}_{\alpha}^{\mathbf{U}} \mathbf{x} \\
\text { s.t. } \mathbf{A}_{\alpha}^{\mathbf{U}} \mathbf{x} \leq \mathbf{b}_{\alpha}^{\mathbf{U}} \\
\mathbf{x} \in \Omega .
\end{gathered}
$$

where all decision variables are non-negative and $\alpha \in(0,1]$.

These problems can be solved by Karush-Kuhn-Tucker's conditions. The optimal solution of original problem (16), $\left(P_{\alpha}\right)$, is inside the solution interval formed by each value of $\alpha \in[0,1]$, and where bounds are obtained by some convex optimization technique in the problems $\left(P_{\alpha}^{L}\right)$ and $\left(P_{\alpha}^{U}\right)$.

This approach, similar as above approach, is limited because it formulates a crisp inequality to compare fuzzy numbers, vector of right-hand sides, with a fuzzy matrix of constraint coefficients. In addition, there is other limitation because it divides the cost matrix with fuzzy elements in two parts which form two quadratic programming problems. One of this problems is formed with the inferior boundaries while the other is formed with the superior ones. However, there not exists a proof that these problems obtain the inferior and superior boundaries of the optimal solution, respectively.

\subsection{Possibilistic Convex Programming}

Some approaches that use possibilistic optimization to solve uncertain convex programming problems will be showed in this subsection. The uncertainties of problems will be described by the possibility theory. Possibilistic programming uses real-valued entities that exist, but the evidence associated with whether or not a particular element belongs to the set is incomplete or hard to obtain. 


\subsubsection{Canestrelli, Giove and Fullér Approach}

Possibilistic quadratic problems with classic variables and imprecise coefficients can be well-posed with small change on possibilistic distribution of objective function when a small change of membership function is provoked. This happens if all $\alpha$-level sets of two vagueness numbers are close to each other then there can be only a small difference between their membership degrees. The application of this approach described in [6] is used in a possibilistic quadratic problem that is defined as:

$$
\begin{gathered}
\min Z=\mathbf{c}^{\mathbf{t}} \mathbf{x}+\mathbf{x}^{\mathbf{t}} \mathbf{Q} \mathbf{x} \\
\text { s.t. } \mathbf{A x} \leq \mathbf{b} \\
\quad \mathbf{x} \in \Omega .
\end{gathered}
$$

where $\tilde{\mathbf{Q}}$ y $\tilde{\mathbf{A}}$ are matrixes with imprecise numbers, $\tilde{\mathbf{c}}$ and $\tilde{\mathbf{b}}$ are vectors with imprecise numbers, and $\Omega$ is a decision variables set in $\mathbb{R}^{n}$. Each imprecise value is associated to a possibilistic distribution.

$\operatorname{Poss}[Z=z]$ is defined as possibilistic distribution of the objective function $\mathrm{Z}$ as developed in the following paragraphs. However, first it is necessary to determine the possibility that $\mathbf{x}$ satisfy the $i$-th constraint which is described as:

$$
\operatorname{Poss}\left[\mathbf{x} \in \mathscr{F}_{i}\right]=\sup _{(\mathbf{A})_{i}, b_{i}}\left\{\Pi\left((\mathbf{A})_{i}, b_{i}\right) \mid(\mathbf{A x})_{i} \leq b_{i}\right\}
$$

where $\Pi\left((\mathbf{A})_{i}, b_{i}\right)=\min \left\{\tilde{a}_{i 1}\left(a_{i 1}\right), \ldots, \tilde{a}_{i n}\left(a_{i n}\right), \tilde{b}_{i}\left(b_{i}\right)\right\},(\mathbf{A})_{i}$ is the marginal possibilistic distribution of $(\tilde{\mathbf{A}})_{i}$, and $b_{i}$ is the marginal possibilistic distribution of $(\tilde{b})_{i}$, for all $i=1, \ldots, m$. Then, for $\mathbf{x} \in \Omega$,

$$
\operatorname{Poss}[\mathbf{x} \in \mathscr{F}]=\min _{i=1, \ldots, m} \operatorname{Poss}\left[\mathbf{x} \in \mathscr{F}_{i}\right]
$$

A conditional possibility, $\operatorname{Poss}[Z=z \mid \mathbf{x}]$, is defined in the second phase. Then, the degree of possibility of objective function is formulated as:

$$
\operatorname{Poss}[Z=z \mid \mathbf{x}]=\sup _{\mathbf{c}, \mathbf{Q}}\left\{\Pi(\mathbf{c}, \mathbf{Q}) \mid \mathbf{c}^{\mathbf{t}} \mathbf{x}+\mathbf{x}^{\mathbf{t}} \mathbf{Q} \mathbf{x}=z\right\}
$$

where $\Pi(\mathbf{c}, \mathbf{Q})=\min _{i, j}\left\{\tilde{c}_{j}\left(c_{j}\right), \tilde{q}_{i j}\left(q_{i j}\right)\right\}$.

Therefore, applying the decision method of Bellman and Zadeh [3], the problem distribution possibilistic is defined as:

$$
\operatorname{Poss}[Z=z]=\sup _{\mathbf{x} \in \Omega} \min \{\operatorname{Poss}[Z=z \mid \mathbf{x}], \operatorname{Poss}[\mathbf{x} \in \mathscr{F}]\} .
$$

Also, this approach formulates a crisp inequality to compare fuzzy numbers, vector of right-hand sides, with a fuzzy matrix of constraint coefficients. 


\subsubsection{Tonon and Bernardini Approach}

An approach to solve fuzzy convex programming with fuzzy costs and fuzzy coefficients in restrictions set is described in [30. Problem (11) can be formulated as a fuzzy convex problem in the following form:

$$
\begin{array}{ll}
\min & f_{0}\left(\mathbf{u}_{0} ; \mathbf{x}\right) \\
\text { s.t. } & f_{i}\left(\mathbf{u}_{i} ; \mathbf{x}\right) \leq \mathbf{0} i=1, \ldots, m \\
& \mathbf{x} \in \Omega .
\end{array}
$$

where $\mathbf{x}$ is a decision variables vector in the feasible solutions set $\Omega, f$ is the objective function, $g_{i}$ are restrictions set functions for each $i=1, \ldots, m$, and $\mathbf{u}_{p}$ are fuzzy parameters vectors for each $p=0,1, \ldots, m$ in the objective function and restrictions.

Vectors $\mathbf{u}_{j}$ can be ordered as:

$$
\mathbf{u}_{j}=\left\{u_{i, 1}, \ldots, u_{i, k_{i}}, u_{\left.i, k_{i}+1, \ldots, u_{i, k_{i}+l_{i}}\right\}}\right.
$$

Non-interactive parameters are in the first $k_{i}$ positions and interactive parameters are in the next $l_{i}$ vector positions [30]. The allowed values of $u_{i, j}, j=1, \ldots, k_{i}$ are restricted by the possibility distribution function $F_{i, j}$. Each dependent parameters vector, $u_{i, j}, j=k_{i}+1, \ldots, k_{i}+l_{i}$, is restricted by a fuzzy relation $F_{i, j}$.

A level $\alpha_{i, j} \in[0,1]$, which is chosen by decision maker, for each vector $\mathbf{u}_{i}$ is selected for $i=0, \ldots, m$ and $j=1, \ldots, k_{i}+l_{i}$, and each vector varies in a $\Psi_{i}$ set:

$$
\Psi_{i}=\left\{F_{i, 1}\left(\alpha_{i, 1}\right) \times \ldots \times F_{i, k_{i}+l_{i}}\left(\alpha_{i, k_{i}+l_{i}}\right)\right\}
$$

where $F_{i, j}\left(\alpha_{i, j}\right)$ marks a cut in the level $\alpha_{i, j}$ of fuzzy set $F_{i, j}$. Thus, Problem (20) can be transformed as:

$$
\begin{array}{ll}
\min _{\mathbf{x}, y} y & \\
\text { s.t. } & \left\{\begin{array}{l}
f_{i}\left(\mathbf{u}_{i} ; \mathbf{x}\right) \leq \mathbf{0} \quad \forall \mathbf{u}_{i} \in \Psi-i, i=1, \ldots, m \\
f_{0}\left(\mathbf{u}_{0} ; \mathbf{x}\right)-y \leq 0 \forall \mathbf{u}_{0} \in \Psi_{0}
\end{array} \mathbf{x} \in \Omega .\right.
\end{array}
$$

\section{Extended Verdegay's Linear Approach ([32])}

As in [9], the constraints of a problem are defined as having a fuzzy nature, that is, some violations in the accomplishment of such restrictions are permitted. In this way, this approach tries to solve the limitations of formulations of the almost last approaches. Therefore if we denote each constraint $\sum_{j \in J} a_{i j} x_{j}$, by $(A x)_{i}$, Problem (2) can be addressed as follows: 


$$
\begin{aligned}
& \min \mathbf{c}^{\mathbf{t}} \mathbf{x}+\frac{1}{2} \mathbf{x}^{\mathbf{t}} \mathbf{Q x} \\
& \text { s.t. }(\mathbf{A x})_{i} \lesssim b_{i}, i \in I \\
& \quad \mathbf{x} \in \Omega
\end{aligned}
$$

where the membership functions:

$$
\mu_{i}: \mathbb{R}^{n} \rightarrow(0,1], \quad i \in I
$$

of the fuzzy constraints are to be determined by decision maker. It is clear that each membership function will give the membership (satisfaction) degree such that any $\mathbf{x} \in \mathbb{R}^{n}$ accomplishes the corresponding fuzzy constraint upon which it is defined. This degree is equal to 1 when the constraint is perfectly accomplished (no violation), and decreases to zero for greater violations. For non-admissible violations the accomplishment degree will equal zero in all cases. In the linear case, these membership functions can be formulated as follows:

$$
\mu_{i}(x)= \begin{cases}1 & (\mathbf{A x})_{i} \leq b_{i} \\ 1-\frac{(\mathbf{A x})_{i}-b_{i}}{d_{i}} & b_{i} \leq(\mathbf{A x})_{i} \leq b_{i}+d_{i} \\ 0 & (\mathbf{A x})_{i}>b_{i}+d_{i}\end{cases}
$$

In order to solve this problem in a two-phase method, as it was shown in 23 , first let us define for each fuzzy constraint, $i \in I$

$$
X_{i}=\left\{\mathbf{x} \in \mathbb{R}^{n} /(\mathbf{A x})_{i} \lesssim b_{i}, \mathbf{x} \in \Omega\right\} .
$$

If $\mathbf{X}=\bigcap_{i \in I} X_{i}$ then last fuzzy quadratic problem can be described as:

$$
\min \left\{\mathbf{c}^{\mathbf{t}} \mathbf{x}+\frac{1}{2} \mathbf{x}^{\mathbf{t}} \mathbf{Q} \mathbf{x} / \mathbf{x} \in \mathbf{X}\right\}
$$

It is clear that $\forall \alpha \in(0,1]$ an $\alpha$-cut of the fuzzy constraint set will be the classical set

$$
X(\alpha)=\left\{\mathbf{x} \in \mathbb{R}^{n} / \mu_{X}(\mathbf{x}) \geq \alpha\right\}
$$

where $\forall x \in \mathbb{R}^{n}$,

$$
\mu_{X}(\mathbf{x})=\min \mu_{i}(\mathbf{x}), i \in I
$$

in which the inf function is used because the fuzzy number can be non-closed set but the $I$ set is finite.

Hence an $\alpha$-cut of the $i$-th constraint will be denoted by $X_{i}(\alpha)$. Therefore, if $\forall \alpha \in(0,1]$,

$$
S(\alpha)=\left\{\mathbf{x} \in \mathbb{R}^{n} / \mathbf{c}^{\mathbf{t}} \mathbf{x}+\frac{1}{2} \mathbf{x}^{\mathbf{t}} \mathbf{Q} \mathbf{x}=\min \mathbf{c}^{\mathbf{t}} \mathbf{y}+\frac{1}{2} \mathbf{y}^{\mathbf{t}} \mathbf{Q} \mathbf{y}, \mathbf{y} \in X(\alpha)\right\}
$$


where the change of the variable is used to find the best solution in the set $X(\alpha)$ formed by $x \in \mathbb{R}^{n}$. The fuzzy solution to the problem will be the fuzzy set defined by the following membership function

$$
S(\alpha)= \begin{cases}\sup \{\alpha: \mathbf{x} \in S(\alpha)\}, & \mathbf{x} \in \bigcup_{\alpha} S(\alpha) \\ 0, & \text { otherwise. }\end{cases}
$$

Provided that $\forall \alpha \in(0,1]$,

$$
X(\alpha)=\bigcap_{i \in I}\left\{x \in \mathbb{R}^{n} /(\mathbf{A x})_{i} \leq r_{i}(\alpha), \mathbf{x} \in \Omega\right\}
$$

with $r_{i}(\alpha)=b_{i}+d_{i}(1-\alpha)$. The operative solution to the former problem can be found, $\alpha$-cut by $\alpha$-cut, by means of the following auxiliary parametric classic programming model,

$$
\begin{array}{ll}
\min & \mathbf{c}^{\mathbf{t}} \mathbf{x}+\frac{1}{2} \mathbf{x}^{\mathbf{t}} \mathbf{Q} \mathbf{x} \\
\text { s.t. } & (\mathbf{A x})_{i} \leq b_{i}+d_{i}(1-\alpha), i \in I \\
\quad & \mathbf{x} \in \Omega, \alpha \in(0,1] .
\end{array}
$$

It is easy to see that the first phase ends when the fuzzy convex programming problem is transformed into several classic convex programming problems. Each one of this problems depends on a parameter which represents the satisfaction level defined by decision maker.

In the second phase the parametric quadratic programming problem is solved for each of the different $\alpha$ values using conventional quadratic programming techniques. We must find solutions to Problem (23) for each $\alpha$ that satisfies Karush-Kuhn-Tucker's necessary and sufficient optimality conditions. One of the conventional techniques is to decide the Lagrange function that is a transformation of Problem (23) in a unconstrained mathematical problem:

$$
L(\mathbf{x}, \mu, v)=\mathbf{c}^{\mathbf{t}} \mathbf{x}+\frac{1}{2} \mathbf{x}^{\mathbf{t}} \mathbf{Q} \mathbf{x}+\mu^{t}(\mathbf{A} \mathbf{x}-\mathbf{b}+\mathbf{d}(1-\alpha))+v^{t}(\mathbf{0}-\mathbf{x})
$$

where $\mu$ and $v$ are the Lagrange multipliers for the inequality and nonnegativity constraints respectively.

Each $\alpha$ is associated to a optimal solution of the parametric convex programming problem and this solutions are called satisfactory solution which generate a set of solutions. Then the Representation Theorem can be used to integrate all these specific $\alpha$-solutions.

Example 1. In order to show the performance of our method, we used the set of historical data shown in Table 1 introduced by Markowitz. The columns 2-10 represent American Tobacco, A.T.\&T., United States Steel, General Motors, Atcheson\&Topeka\&Santa Fe, Coca-Cola, Borden, Firestone and Sharon Steel securities data, respectively. The returns on the nine securities, during the years 1937-54, are presented in Table 1 . 
Table 1 Fuzzy portfolio selection problem

\begin{tabular}{|c|c|c|c|c|c|c|c|c|}
\hline$\# 1$ & $\neq 2$ & $\overline{\# 3}$ & $\# 4$ & $\# 5$ & $\# 6$ & $\# 7$ & $\# 8$ & \#9 \\
\hline $\operatorname{arar} \mathrm{Ar}$ & A T T\& & $\mathrm{SS}$ & G.M & $\Gamma 8$ & C.C. & $\mathrm{dm}$ & rst & S.S. \\
\hline $77-0.30$ & 173 & .318 & 477 & 57 & .065 & -0.319 & -0.4 & -0.43 \\
\hline $\begin{array}{ll}8 & 0.51\end{array}$ & 8 & 5 & 0.714 & 7 & 38 & 0.076 & 0.336 & 0.238 \\
\hline 390.055 & 0.2 & 7 & 0.165 & 4 & .078 & 0.381 & -0.09 & .29 \\
\hline 0 & 03 & 4 & 3 & 9 & -0.077 & -0.001 & 0.09 & -0.03 \\
\hline 2 & -0.183 & -0.171 & 7 & 7 & 37 & 87 & 0 & • \\
\hline 0.0 & 0.067 & .039 & 0.476 & 0.865 & 56 & 0.262 & 1.113 & 0.126 \\
\hline 30 & 0 & 19 & U & 5 & 0.351 & 0.341 & 0.580 & 0.63 \\
\hline $\begin{array}{ll}4 & 0.19\end{array}$ & 03 & 0.260 & 0 & 7 & 33 & 0.227 & 0.473 & 0.28 \\
\hline $\begin{array}{ll}5 & 0.44\end{array}$ & 0.216 & .419 & 0.216 & 0.373 & 0.349 & 0.352 & 0.229 & 0.578 \\
\hline $6-0.088$ & -0.046 & -0.078 & -0.272 & -0.037 & -0.209 & 0.153 & -0.126 & 0.28 \\
\hline 12 & -0.071 & 0.169 & 0.144 & 0.026 & 0.355 & -0.099 & 0.009 & 0.18 \\
\hline O & 0.056 & -0.035 & 0.107 & 0.153 & -0 & 0.0 & 0 & 0.11 \\
\hline 90.305 & 0.030 & 0.133 & 0.321 & 7 & 0.246 & 0.273 & 0.223 & רי 0. \\
\hline $1950-0.096$ & 0 & 0 & 0 & 0.579 & -0.248 & 0.091 & 0.650 & 0.3 \\
\hline 0 & 0 & .021 & 0 & 0.040 & -0. & 0. & -0 & 0.3 \\
\hline 0.12 & 0 & 0.131 & 0 & 0 & 0.079 & 0.109 & 0.175 & 0.0 \\
\hline-0.0 & 0.035 & 0.006 & -0.072 & $-0 .($ & 0.067 & 0.21 & -0. & -0 \\
\hline 19540.15 & 176 & 908 & 0.715 & 0.469 & 0.077 & 0.112 & 0.756 & 0.185 \\
\hline
\end{tabular}

This example will consider performances of portfolios with respect to "return" thus defined. This assumes that a dollar of realized or unrealized capital gains is exactly equivalent to a dollar of dividends, no better and no worse. This assumption is appropriate for certain investors, for example, some types of tax-free institutions. Other ways of handling capital gains and dividends, which are appropriate for other investors, can be viewed in 20.

Here we show the results obtained for the porfolio selection problem, described by Table 1 by the fuzzy quadratic programming methods introduced in this section and its solution is shown in Table 2, By computing the average value of all the years of each column of random variables of Table 1, we obtained the expected values of each return of the securities. 
Table 2 Results of the first phase of the portfolio selection problem

\begin{tabular}{|c|c|c|c|c|}
\hline$\alpha$ & $\mathbf{x}$ & Solution & Time \\
\hline \hline 0.0 & {$[-0.0000 ; 0.1236 ; 0.1374 ; 0.0000 ; 0.0910 ; 0.0641 ; 0.5838 ; 0.0000 ; 0.0000]$} & 0.0235 & 1.0313 \\
0.1 & {$[0.0000 ; 0.0698 ; 0.1509 ; 0.0000 ; 0.0924 ; 0.0610 ; 0.6259 ; 0.0000 ;-0.0000]$} & 0.0247 & 0.1563 \\
0.2 & {$[0.0000 ; 0.0303 ; 0.1713 ; 0.0000 ; 0.0936 ; 0.0465 ; 0.6584 ; 0.0000 ;-0.0000]$} & 0.0259 & 0.1250 \\
0.3 & {$[0.0000 ;-0.0000 ; 0.1768 ; 0.0056 ; 0.0967 ; 0.0251 ; 0.6958 ; 0.0000 ;-0.0000]$} & 0.0272 & 0.1563 \\
0.4 & {$[0.0000 ;-0.0000 ; 0.1627 ; 0.0306 ; 0.1168 ;-0.0000 ; 0.6899 ;-0.0000 ; 0.0000]$} & 0.0287 & 0.1406 \\
0.5 & {$[0.0000 ;-0.0000 ; 0.1323 ; 0.0736 ; 0.1553 ;-0.0000 ; 0.6388 ;-0.0000 ; 0.0000]$} & 0.0307 & 0.1563 \\
0.6 & {$[-0.0000 ;-0.0000 ; 0.0975 ; 0.1224 ; 0.1912 ; 0.0000 ; 0.5889 ; 0.0000 ; 0.0000]$} & 0.0332 & 0.1250 \\
0.7 & {$[-0.0000 ;-0.0000 ; 0.0703 ; 0.1554 ; 0.2354 ; 0.0000 ; 0.5389 ;-0.0000 ; 0.0000]$} & 0.0364 & 0.1250 \\
0.8 & {$[0.0000 ; 0.0000 ; 0.0368 ; 0.2002 ; 0.2735 ; 0.0000 ; 0.4894 ; 0.0000 ;-0.0000]$} & 0.0402 & 0.1250 \\
0.9 & {$[0.0000 ;-0.0000 ; 0.0048 ; 0.2422 ; 0.3131 ; 0.0000 ; 0.4399 ; 0.0000 ;-0.0000]$} & 0.0445 & 0.1250 \\
1.0 & {$[-0.0000 ;-0.0000 ;-0.0000 ; 0.2717 ; 0.3537 ; 0.0000 ; 0.3746 ;-0.0000 ; 0.0000]$} & 0.0495 & 0.1250 \\
\hline
\end{tabular}

\section{Conclusions}

Fuzzy Convex Programming problems are of utmost importance in an increasing variety of practical fields, but unfortunately little study has been done with this important class of problems.

This paper shows a general view about fuzzy convex mathematical programming and some known methods that were developed to solve convex problems with vagueness in different parts are described. Tang and Wang's approach can transform a flexible programming problem into two classical problems. The transformed problems have different constraints where are limited by $\alpha$-levels which may be defined by decision maker or used as objective function of the classical problem. However this approach is limited because it formulates a comparison of fuzzy numbers, vector right-hand sides, with a crisp matrix of constraint coefficients. Liu's and, Ammar and Khalifa's approaches transform flexible programming problem into two classical programming problems that determine the superior and inferior boundaries to each $\alpha$-level chosen by decision maker. Thus, this interval is a solution set to the original flexible programming problem but there is not a proof that these problems obtain the inferior and superior boundaries of the optimal solution, respectively.

The limitations of formulations of the presented approaches are solved with the extension of Verdegay's linear approach because our approach is not necessary to choose $\alpha$-level. Therefore, this approach can be used as a general method to solve convex programming problems with uncertainties in the set of constraints.

In addition, there are other approaches that deal with the uncertain data in optimization problems. For example, we showed two approaches that use the possibilistic programming which represents imprecise data by possibility distributions. In this case, the real-valued exist, but it may be or not to belong to the set is incomplete or hard to obtain. However, we cannot take a match of these approaches because they model the uncertainties of different way. 
Acknowledgements. The authors want to thank the support provided by the Brazilian agency CAPES and the Spanish projects TIN2008-06872-C04-04, TIN2008-01948, P07-TIC-02970 and 04552/GERM/06.

\section{References}

1. Ammar, E., Khalifa, H.A.: Fuzzy portfolio optimization a quadratic programming approach. Chaos, Solitons and Fractals 18, 1045-1054 (2003)

2. Bector, C.R., Chandra, S.: Fuzzy mathematical programming and fuzzy matrix games. Studies in Fuzziness and Soft Computing, vol. 169. Springer, Berlin (2005)

3. Bellman, R.E., Zadeh, L.A.: Decision-marking in a fuzzy environment. Management Science 17(4), B141-B164 (1970)

4. Bertsekas, D.P., Nedié, A., Azdaglar, A.E.: Convex analysis and optimization. Athena Scientific, Belmont (2003)

5. Boyd, S., Vandenberghe, L.: Convex optimization. Cambridge University Press, Cambridge (2004)

6. Canestrelli, E., Giove, S., Fullér, R.: Stability in possibilistic quadratic programming. Fuzzy Sets and Systems 82, 51-56 (1996)

7. Chen, Y.S.: Fuzzy ranking and quadratic fuzzy regression. Computers and Mathematics with Applications 38, 265-279 (1999)

8. Donoso, S., Marín, N., Vila, M.A.: Fuzzy Regression with Quadratic Programming: An Application to Financial Data. In: Corchado, E., Yin, H., Botti, V., Fyfe, C. (eds.) IDEAL 2006. LNCS, vol. 4224, pp. 1304-1311. Springer, Heidelberg (2006)

9. Delgado, M., Verdegay, J.L., Vila, M.A.: A general model for fuzzy linear programming. Fuzzy Sets and Systems 29, 21-29 (1989)

10. Dubois, D., Prade, H.: Fuzzy sets and systems: theory and applications. Academic Press, San Diego (1980)

11. Dubois, D., Prade, H.: Possibility theory: an approach to computerized processing of uncertain. Plenum Press, New York (1988)

12. Inuiguchi, M., Ramik, J.: Possibilistic linear programming: A brief review of fuzzy mathematical programming and a comparison with stochastic programming in portfolio selection problem. Fuzzy Sets and Systems 111(1), 3-28 (2000)

13. Kim, E., Kang, H.J., Park, M.: Numerical stability analysis of fuzzy control systems via quadratic programming and linear matrix inequalities. IEEE Transactions on Systems, Man and Cybernetics - Part A: Systems and Humans 29(4), 333-346 (1999)

14. Klir, G.: Where do we stand on measures of uncertainty, ambiguity, fuzziness, and the like. Fuzzy Sets and Systems 24, 141-160 (1987)

15. Lai, Y.J., Hwang, C.L.: Fuzzy mathematical programming: methods and applications. Lecture notes in economics and mathematical systems, vol. 394. Springer, Berlin (1992)

16. León, T., Liern, V., Vercher, E.: Viability of infeasible portfolio selection problems: A fuzzy approach. European Journal of Operational Research 139, 178189 (2002)

17. Liu, S.T.: Quadratic programming with fuzzy parameters: A membership function approach. Chaos, Solitons and Fractals (2007) (Article in press) 
18. Liu, S.T.: Solving quadratic programming with fuzzy parameters based on extension principle. In: IEEE International Conference Fuzzy Systems, FUZZIEEE 2007, London, UK, pp. 1-5 (2007)

19. Lodwick, W.A., Jamison, K.D.: Theoretical and semantic distinctions of fuzzy, possibilistic, and mixed fuzzy/possibilistic optimization. Fuzzy Sets and Systems 158, 1861-1872 (2007)

20. Markowitz, H.M.: Portfolio Selection: Efficient Diversification of Investments, 2nd edn. Blackwell Publisher, Massachusetts (1991)

21. Mollov, S., Babuska, R., Abonyi, J., Verbruggen, H.B.: Effective optimization for fuzzy model predictive control. IEEE Transactions on Fuzzy Systems 12(5), 661-675 (2004)

22. Negoita, C.V., Ralescu, D.A.: Applications of fuzzy sets to systems analysis. Birkhauser Verlag, Stuttgard (1975)

23. Silva, R.C., Verdegay, J.L., Yamakami, A.: Two-phase method to solve fuzzy quadratic programming problems. In: IEEE International Conference Fuzzy Systems, FUZZ-IEEE 2007, London, UK, pp. 1-6 (2007)

24. Tanaka, H., Guo, P., Türksen, B.: Portfolio selection based on fuzzy probabilities and possibility distributions. Fuzzy Sets and Systems 111, 387-397 (2000)

25. Tanaka, H., Lee, H.: Interval regression analysis by quadratic programming approach. IEEE Transactions on Fuzzy Systems 6(4), 473-481 (1998)

26. Tang, J., Wang, D.: An interactive approach based on a genetic algorithm for a type of quadratic programming problems with fuzzy objective and resources. Computers \& Operations Research 24(5), 413-422 (1997)

27. Tang, J., Wang, D.: A nonsymmetric model for fuzzy nonlinear programming problms with penalty coefficients. Computers \& Operations Research 24(8), 717-725 (1997)

28. Tang, J., Wang, D., Fung, R.Y.K.: Fuzzy formulation for multi-product aggregate production planning. Production, Planning \& Control 11(7), 670-676 (2000)

29. Tankeh, A., Mandani, H.: Convex fuzzy controller: neuro-fuzzy and convex optimisation. In: Sixth IEEE International Conference on Fuzzy Systems, pp. 1133-1139 (1997)

30. Tonon, F., Bernardini, A.: A random set approach to the optimizatino of uncertain structures. Computers and Structures 68, 583-600 (1998)

31. Vercher, E., Bermúdez, J., Segura, J.: Portfolio selection based on on fuzzy probability and possibility distributions. Fuzzy Sets and Systems 158(7), 769$782(2007)$

32. Verdegay, J.L.: Fuzzy mathematical programming. In: Gupta, M.M., Sanches, E. (eds.) Fuzzy Information and Decision Procsses, pp. 231-237. North-Holland Publishing Company, Amsterdam (1982)

33. Verdegay, J.L., Yager, R.R., Bonissone, P.P.: On heuristics as a fundamental constituent of soft computing. Fuzzy Sets and Systems 159(7), 846-855 (2008)

34. Wang, S., Zhu, S.: On fuzzy portfolio selection problems. Fuzzy Optimization and Decision Making 1, 361-377 (2002)

35. Watada, J.: Fuzzy portfolio selection and its applications to decision making. Tatra Mountains Mathematics Publication 13, 219-248 (1997)

36. Xu, Z., Da, Q., Chen, Q.: Priority approach based on quadratic programming model to fuzzy preference relation. Journal of Southeast University (English Edition) 21(1), 108-110 (2005)

37. Zimmermann, H.: Applications of fuzzy sets theory to mathematical programming. Information Sciences 36, 29-58 (1985) 